\title{
Reconstruction of the pulmonary artery for lung cancer: Long-term results
}

\author{
Federico Venuta, MD, ${ }^{\mathrm{a}}$ Anna Maria Ciccone, MD, ${ }^{\mathrm{b}}$ Marco Anile, MD, ${ }^{\mathrm{a}}$ Mohsen Ibrahim, MD, \\ Tiziano De Giacomo, MD, ${ }^{\mathrm{a}}$ Giorgio F. Coloni, MD, ${ }^{\mathrm{a}}$ and Erino A. Rendina, MD ${ }^{\mathrm{b}}$
}

\begin{abstract}
Objective: Reconstruction of the pulmonary artery in association with lung resection is technically feasible with low morbidity and mortality. To assess long-term outcome, we report our 20-year experience.

Methods: Between 1989 and 2008, we performed pulmonary artery reconstruction in 105 patients with nonsmall cell lung cancer (tangential resections not included). Twenty-seven patients received induction therapy. We performed 47 pulmonary artery sleeve resections, 55 reconstructions by pericardial patch (with 3 left pneumonectomies under cardiopulmonary bypass), and 3 by pericardial conduit. In 65 patients, a bronchial sleeve resection was associated; in 6 cases superior vena caval reconstruction was also required. Fifteen patients had stage IB disease, 37 stage II, 31 IIIA, and 22 IIIB. Sixty-one patients had epidermoid carcinoma, and 38 adenocarcinoma. Mean follow-up was $46 \pm 40$ months.
\end{abstract}

Results: The procedure-related complications were 1 pulmonary artery thrombosis requiring completion pneumonectomy and 1 massive hemoptysis leading to death (operative mortality, $0.95 \%$ ); 28 patients had other complications, with the most frequent prolonged air leakage. Overall 5-year survival was $44 \%$. Five- and 10 -year survivals for stages I and II versus stage III were, respectively, $60 \%$ versus $28 \%$ and $25 \%$ versus $12 \%$. Fiveyear survivals were $52.6 \%$ for N0 and N1 nodal involvement versus $20 \%$ for N2; 10 -year survivals were $28 \%$ versus $3 \%$. Multivariate analysis yielded induction therapy, N2 status, adenocarcinoma, and isolated pulmonary artery reconstruction as negative prognostic factors.

Conclusions: Pulmonary artery reconstruction is safe, with excellent long-term survival. Our results support this technique as an effective option for patients with lung cancer.

Resection and reconstruction of the pulmonary artery (PA) is considered feasible and safe for patients with non-small cell lung cancer (NSCLC). This procedure is usually indicated for tumors or satellite lymph nodes that involve the PA at the lobar hilum and preclude complete resection by simple lobectomy. The artery can be involved to various degrees, from partial infiltration to a more extensive and even circumferential invasion, although endovascular growth of the tumor is rare. This heterogeneous presentation may require either patch reconstruction, end-to-end anastomosis, or interposition of a prosthetic conduit; simple tangential resection with direct suture traditionally is not included in the domain of PA reconstruction by our group. The choice of the most appropriate technique of reconstruction is usually made at the time of surgery on the basis of the extent of vascular involvement.

\footnotetext{
From the Department of Thoracic Surgery, ${ }^{\text {a }}$ Policlinico Umberto I, University of

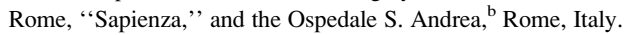

Read at the Eighty-ninth Annual Meeting of The American Association for Thoracic Surgery, Boston, Mass, May 9-13, 2009.

Received for publication May 14, 2009; revisions received July 10, 2009; accepted for publication July 20, 2009; available ahead of print Sept 10, 2009.

Address for reprints: Federico Venuta, MD, Cattedra di Chirurgia Toracica, Policlinico Umberto I, Università di Roma "Sapienza," Via le del Policlinico 155, 00166 Rome, Italy (E-mail: federico.venuta@uniroma1.it).

J Thorac Cardiovasc Surg 2009;138:1185-91

$0022-5223 / \$ 36.00$

Copyright $(C) 2009$ by The American Association for Thoracic Surgery

doi:10.1016/j.jtcvs.2009.07.043
}

This surgical technique was initially advocated only when pulmonary function is so compromised to preclude pneumonectomy; in fact, at that time, the latter was the only procedure considered appropriate to achieve cure in patients with proximal lesions. It is now clear, however, that pneumonectomy adds increased risk, ${ }^{1}$ especially when performed on the right side and after induction chemotherapy, ${ }^{2}$ with deranged cardiac function, ${ }^{3}$ worse quality of life, ${ }^{4}$ and decreased survival, particularly at early stages and in elderly patients. ${ }^{5,6}$ These considerations have led to a better evaluation of the potentials of reconstructive procedures of the bronchus and PA associated with lobectomy. It is now clear that bronchial sleeve resection is oncologically comparable to pneumonectomy, with the advantage of preserving pulmonary function and decreasing morbidity and mortality. ${ }^{7-10}$ On the vascular side, however, reconstructive procedures of the PA have encountered more difficulty in gaining acceptance because of historical prejudices and lack of studies on large and homogeneous series of patients with longterm follow up. We therefore report here our 20-year experience with PA reconstruction alone or in association with bronchial sleeve resection.

\section{MATERIALS AND METHODS}

From 1989 to 2008 , we performed PA reconstruction on 105 consecutive patients. The study was approved by the Institutional Review Board of the University "Sapienza" of Rome. During this time, we performed a mean of 


\section{Abbreviations and Acronyms \\ $\mathrm{CPB}=$ cardiopulmonary bypass \\ $\mathrm{CT}=$ computed tomography \\ NSCLC $=$ non-small cell lung cancer \\ PA $=$ pulmonary artery}

$5.25 \pm 1.9$ PA reconstructions per year. This procedure accounted for a mean of $3.6 \% \pm 2.1 \%$ of the resections performed at our institution for lung cancer; during the same period, 187 pneumonectomies were performed (9.3 \pm 4.9 per year). Patients undergoing PA reconstruction before 1989 were not included in this study because they do not represent a homogeneous population in terms of intraoperative and postoperative management. Tangential resections of the PA were not included in this series because they have not been considered as vascular reconstructions but rather as a simple variation of standard lobectomy. Preoperative workup included total body contrast CT, pulmonary function tests, blood gas analysis, cardiac evaluation, and basic examinations as usual. Magnetic resonance imaging and positron emission tomography were performed in selected cases. Patients who were anticipated to be able to tolerate pneumonectomy according to standard preoperative evaluation criteria were not excluded from consideration for PA reconstruction. Patients who had biopsy-proven N2 disease received neoadjuvant chemotherapy with a cisplatin-based regimen and were subsequently reassessed. Preoperative radiotherapy is not routinely used at our center. The surgical technique for PA reconstruction and bronchial sleeve lobectomy has been previously described ${ }^{11,12}$ and was uniform throughout the study period. The only intraoperative management modification was related to intravenous heparin administration: from the initial 3000 to 5000 units previously reported, ${ }^{11}$ we moved to a dose of 1500 to 2000 units. Operative morbidity and mortality were considered any event within 30 days of surgery or during the same hospitalization. No patients were unavailable for follow-up. The mean follow-up was $46 \pm 40$ months (range 1-180 months); median follow-up was 32 months.

Patients were followed up after surgery with serial chest radiographs for the first 6 months. Contrast total body CT was performed every 6 months for 2 years and then yearly. Positron emission tomography with fludeoxyglucose F 18 (INN fludeoxyglucose $\left[\mathrm{F}^{18}\right]$ ) has been included more recently in selected cases.

Data were collected and stored with an Excel data base (Microsoft Corp, Redmond, Wash). Quantitative variables were expressed as mean \pm SD, whereas nominal variables were expressed binarily as presence (1) or absence $(0)$ of the event. In the univariate analysis, qualitative variables were analyzed with the Pearson $\chi^{2}$ test or Fisher exact test as required. Multivariate analysis was performed by Cox regression on variables that showed statistical significance at univariate analysis. Survival curves were plotted with the Kaplan-Meier formula; the log-rank test was performed to compare survival between different groups. The statistical analysis was performed with the STATISTICA data analysis software system (version 7; StatSoft Inc, Tulsa, Okla).

\section{RESULTS}

There were 87 men $(83 \%)$ and 18 women $(17 \%)$. The mean age was $62 \pm 10.5$ years. The mean preoperative forced expiratory volume in 1 second was $76.1 \% \pm 14 \%$ predicted (median, $75.5 \%$; range, $41 \%-104 \%$ ). Seventyeight percent of the patients were current or former smokers. Comorbidities were present in 36 patients $(34.3 \%)$. The most common were arterial hypertension (18 patients, $17 \%$ ) and chronic obstructive pulmonary disease ( 8 patients, $8 \%$ ); other comorbidities were previous myocardial infarction (6 patients, 17\%), diabetes (4 patients, 11\%), gastric ulcer ( 2 patients, $6 \%$ ), carotid stenosis ( 1 patient, $3 \%$ ), cerebrovascular problems ( 2 patients, $3 \%$ ), and favism (1 patient, $3 \%$ ). Induction chemotherapy was administered in 23 patients $(22 \%)$, whereas preoperative radiotherapy was performed only in $4(4 \%)$; all patients receiving induction were in stage III, with the exception of 3 patients referred to our center after 3 cycles of chemotherapy administered elsewhere who were in stage IIB. Forty-two patients $(40 \%)$ received adjuvant therapy. Seventy-four procedures $(70.5 \%)$ were performed on the left side and 31 $(29.5 \%)$ on the right. We performed 47 PA sleeve resections $(44.8 \%), 55(52.3 \%)$ reconstructions by a pericardial patch (3 associated with left pneumonectomy under cardiopulmonary bypass $[\mathrm{CPB}])$, and $3(2.9 \%)$ by interposition of a pericardial conduit. Patches were made with autologous pericardium in 47 patients $(85.5 \%)$ and with bovine pericardium (Synovis Inc, Minneapolis, Minn) in 8 (14.5\%). All pericardial conduits were required on the left side; they were made with autologous pericardium in 2 cases and bovine pericardium in 1 and were prepared with the technique previously described. ${ }^{12}$ The 3 patients undergoing pneumonectomy and patch reconstruction under $\mathrm{CPB}$ received induction radiotherapy. In 65 patients $(62 \%)$, PA reconstruction was associated with bronchial sleeve resection; in all other cases it was associated with standard lobectomy, with the exception of 1 patient undergoing bilobectomy. The combined bronchovascular reconstructions were performed on the left side in 45 cases $(69.2 \%)$ and on the right in $20(30.7 \%)$. All patients underwent upper lobectomy (left upper lobectomy most frequently at 72 patients, $69 \%$ ), with the exception of 2 who required left lower sleeve lobectomy with reimplantation of the upper lobe. In all patients who also underwent bronchial sleeve resection, an intercostal muscle flap was wrapped around the bronchial anastomosis to protect and revascularize the suture line and act as a buffer between the vascular and bronchial repairs. In 6 cases superior vena cava reconstruction was also required: in 5 cases the vessel was reconstructed with a graft ( 2 polytetrafluoroethylene and 3 bovine pericardium tube) and in 1 case with an autologous pericardium patch. Five of these patients required a combined bronchovascular reconstruction. One patient also underwent en bloc resection of the chest wall. Fifteen patients $(14.7 \%)$ were in stage IB, $37(35.3 \%)$ were in stage II (7 IIA and 30 IIB), 31 (29.5\%) were in stage IIIA, and $22(20.9 \%)$ were in stage IIIB. Sixty-one patients $(58 \%)$ had epidermoid carcinoma, $38(36.2 \%)$ had adenocarcinoma, $1(0.9 \%)$ had mixed, and $5(4.8 \%)$ had large cell. One hundred one patients $(96 \%)$ underwent complete resection; in the last 53 procedures, only 1 patient $(1.9 \%)$ underwent incomplete resection.

Overall morbidity was $28.5 \%$ (30 patients). The procedure-related major complications were $1 \mathrm{PA}$ thrombosis requiring completion pneumonectomy (postoperative day 
2, occlusion of left PA demonstrated by angiography) and 1 massive hemoptysis leading to death (postoperative day 28); the latter complication occurred in a patient undergoing combined bronchovascular reconstruction. The operative mortality was $0.95 \%$. Twenty-eight patients $(27 \%)$ had other complications. The most frequent was prolonged air leak (10 patients, 9.5\%, 3 cases after induction chemotherapy); other complications were atrial fibrillation (5 patients, 2 of whom had undergone patch reconstruction and pneumonectomy under CPB), empyema (3 patients, 2 after induction chemotherapy), bronchopleural fistula (1 patient), respiratory failure requiring noninvasive respiratory support (1 patient), recurrent nerve paralysis (1 patient), pleural effusion (1 patient), abdominal perforation and sepsis (1 patient), and wound-related problems (5 patients). Overall, only 9 complications $(30 \%)$ occurred in patients receiving induction therapy, without any statistically significant difference $(P=.9)$. No PA obstruction or evidence of decreased flow was detected with contrast $\mathrm{CT}$ performed during follow-up.

Patients undergoing pneumonectomy and patch reconstruction under CPB survived 35 days (pneumonia after discharge) and 25 and 36 months (both distant recurrences). Because of the rarity of this procedure, these 3 patients were excluded from survival analysis, which was therefore performed on the remaining 102 patients.

Overall 5- and 10-year survivals were $44 \%$ and $20 \%$, respectively (Figure $1, A$ ). The disease-free survivals (no recurrence) were $43.1 \%$ at 5 years and $30.2 \%$ at 10 years (Figure $1, B)$. Patients in stage IB had 5- and 10-year survivals of $75 \%$ and $44 \%$ respectively; other survivals were $53 \%$ and $21 \%$ for stage II, $31 \%$ and $17 \%$ for stage IIIA, and $19 \%$ and $6 \%$ for stage IIIB. There were no statistically significant differences between stages IB and II $(P=.3)$ or between stages IIIA and IIIB $(P=.5)$; for this reason, these stages were considered in 2 groups (stages I and II vs stages IIIA and IIIB) in the survival analysis. The 5- and 10-year survivals for stage I and II were $60 \%$ and $28 \%$, respectively; those for stage III were $25 \%$ and $12 \%$ (stages I and II vs stages IIIA and IIIB, $P=.004$ ). The 5 - and 10 -year survivals for squamous and nonsquamous carcinomas were $59.7 \%$ versus $20.2 \%$ and $25 \%$ versus $13 \%$. The $\mathrm{N}$ status had a significant effect on survival: patients with N0 disease had 5- and 10-year survivals of $67 \%$ and $33 \%$, respectively. For N1 disease, the 5- and 10-year survivals were $46 \%$ and $26 \%$; for $\mathrm{N} 2$, they were $20 \%$ and $3 \%$. There was no statistical difference in survival between $\mathrm{N} 0$ and $\mathrm{N} 1$ disease at any point $(P=.5)$. For this reason, the 2 categories of N0 and N1 have been considered together in the survival analysis (Figure 2), demonstrating the negative effect of N2 status. There were 6 local recurrences $(5.7 \%)$ and 40 distant recurrences $(38 \%)$. Stage I and II lesions did not recur locally. The 5-year survivals of patients undergoing isolated PA reconstruction and combined bronchovascular reconstruction were $23 \%$ and $55, \%$ respectively; at 10 years, they were
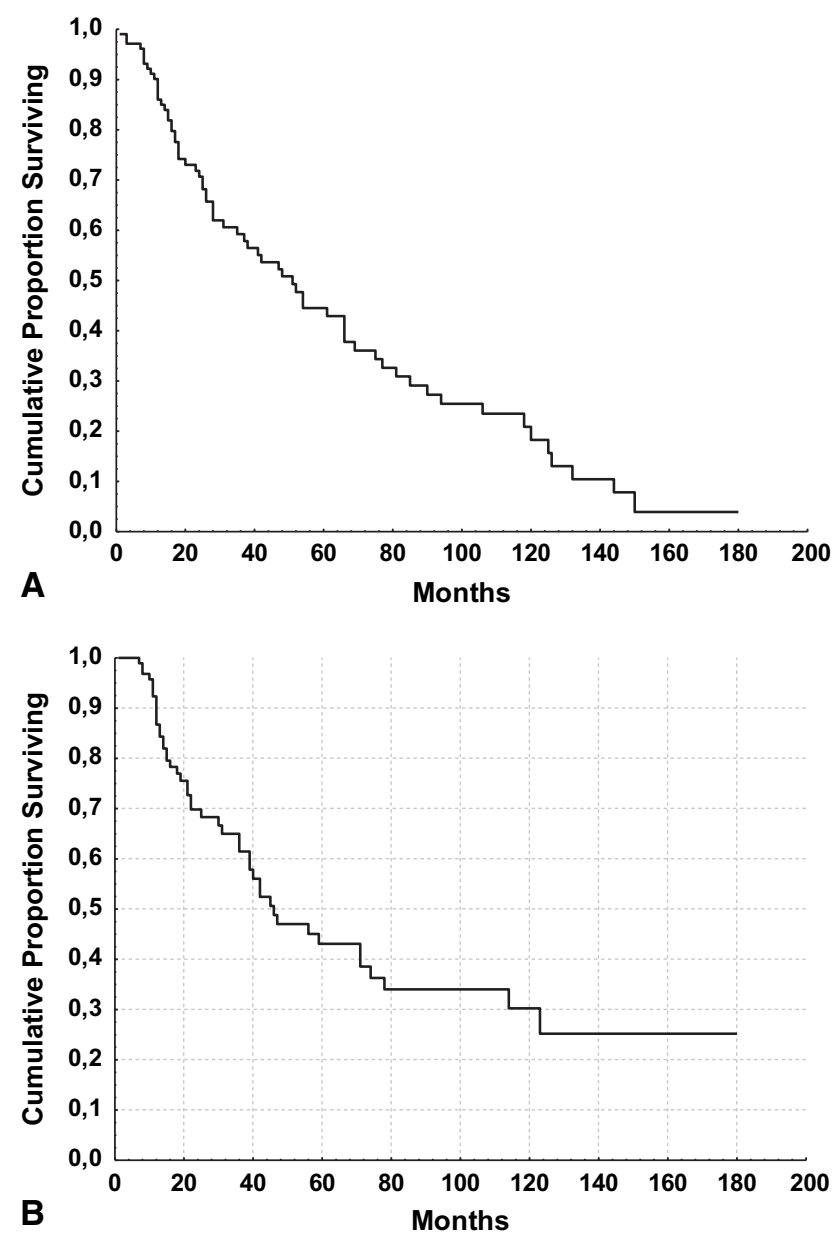

FIGURE 1. A, Overall survival. B, Disease-free survival.

$7 \%$ and $30 \%$ (isolated PA vs combined $P=.01$ ). Disease-free survivals for the same categories at 5 and 10 years were respectively $45 \%$ versus $43 \%$ and $45 \%$ versus $27 \%$ $(P=.9)$. Univariate analysis of overall survival (Table 1$)$ showed significant differences for stages I and II versus stage III, induction versus noninduction therapy, PA reconstruction alone versus combined bronchovascular reconstruction, squamous versus nonsquamous histologic type, $\mathrm{N} 0$ and $\mathrm{N} 1$ versus N2, and presence of complications and recurrence versus none. A trend toward significance was seen for patch reconstruction versus sleeve resection. At multivariate analysis (Table 2), only the diagnosis of adenocarcinoma, N2 disease, administration of induction therapy, onset of recurrence, and isolated PA reconstruction had confirmed negative effects on outcome. Univariate and multivariate analyses were repeated for disease-free survival; in those analyses, isolated PA reconstruction did not show a negative effect.

\section{DISCUSSION}

Complete surgical resection remains the cornerstone for long-term survival of patients with NSCLC. With this intent, 


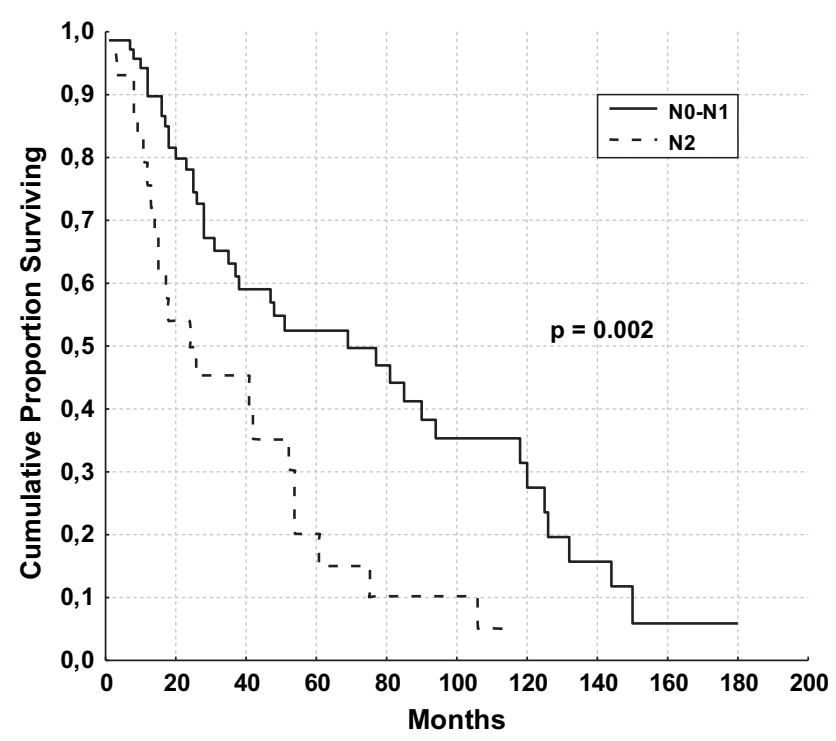

FIGURE 2. Survival by N status.

pneumonectomy, when functionally feasible, has been considered for several decades to be the standard treatment for central or locally advanced lesions. It is now clear, however, that this operation entails increased morbidity and mortality and is associated with a worse outcome. It should therefore be performed only when no lesser resection is technically feasible. This assumption has helped bronchial sleeve resection to gain progressively a stable position in the surgical armamentarium. A number of studies initially supported its feasibility and safety ${ }^{13}$ and subsequently also demonstrated its functional and survival advantages relative to pneumonectomy. ${ }^{7-10}$ A recent meta-analysis ${ }^{8}$ demonstrated that parenchyma-sparing operations may reduce by $30 \%$ the death risk relative to pneumonectomy and thus increase survival, especially for patients with stages I and II NSCLC.

Despite the acceptance of bronchial reconstruction as an oncologically reliable alternative to pneumonectomy independent of the functional status of the patient, on the vascular side the concept of PA reconstruction has encountered more difficulties in gaining full acceptance. The potential risks were initially overestimated because of historical concern with this unfamiliar situation; the involvement of a low-pressure system might hypothetically favor the onset of catastrophic intraoperative and postoperative complications. The supposed requirement of full-dose heparinization, as for other vascular procedures involving the systemic circulation, might predispose toward hemorrhage or alternatively to thrombosis of the anastomosis. The initial reports ${ }^{14-17}$ did not alleviate this concern; on the contrary, this feeling was even exacerbated by the high rate of PA thrombosis when tangential resection was performed. ${ }^{16,17}$ Major concerns were thus initially related to feasibility, safety, long-term patency, and effect on cardiopulmonary function; only later did oncologic reliability and long-term survival come to the fore. With
TABLE 1. Univariate analysis for mortality

\begin{tabular}{|c|c|c|c|c|c|c|c|}
\hline \multirow[b]{2}{*}{ Variable } & \multicolumn{2}{|c|}{ Total } & \multicolumn{2}{|c|}{ Alive } & \multicolumn{2}{|c|}{ Dead } & \multirow[b]{2}{*}{$P$ value } \\
\hline & No. & $\%$ & No. & $\%$ & No. & $\%$ & \\
\hline Sex & & & & & & & .9 \\
\hline Male & 86 & $84.3 \%$ & 33 & $32.3 \%$ & 53 & $52 \%$ & \\
\hline Female & 16 & $15.7 \%$ & 6 & $5.9 \%$ & 10 & $9.8 \%$ & \\
\hline Age & & & & & & & .5 \\
\hline$\geq 65 \mathrm{y}$ & 51 & $50 \%$ & 18 & $17.6 \%$ & 33 & $32.3 \%$ & \\
\hline$<65 \mathrm{y}$ & 51 & $50 \%$ & 21 & $20.7 \%$ & 30 & $29.4 \%$ & \\
\hline Stage & & & & & & & .03 \\
\hline I or II & 52 & $51 \%$ & 25 & $24.5 \%$ & 27 & $26.4 \%$ & \\
\hline III & 50 & $49 \%$ & 14 & $13.7 \%$ & 36 & $35.2 \%$ & \\
\hline Induction & & & & & & & .003 \\
\hline Yes & 24 & $23.5 \%$ & 3 & $2.9 \%$ & 21 & $20.7 \%$ & \\
\hline No & 78 & $76.5 \%$ & 36 & $35.3 \%$ & 42 & $41 \%$ & \\
\hline Reconstruction techniq & que* & & & & & & .05 \\
\hline Patches & 52 & $52.5 \%$ & 16 & $16.1 \%$ & 36 & $36.4 \%$ & \\
\hline Sleeves & 47 & $47.5 \%$ & 22 & $22.2 \%$ & 25 & $25.3 \%$ & \\
\hline Procedure & & & & & & & .009 \\
\hline PA alone & 37 & $36 \%$ & 8 & $7.8 \%$ & 29 & $28.4 \%$ & \\
\hline $\begin{array}{l}\text { PA with } \\
\text { bronchial sleeve }\end{array}$ & 55 & $67 \%$ & 31 & $30.4 \%$ & 34 & $33.4 \%$ & \\
\hline Complications & & & & & & & .007 \\
\hline Yes & 30 & $29.4 \%$ & 6 & $5.9 \%$ & 24 & $23.5 \%$ & \\
\hline No & 72 & $70.6 \%$ & 33 & $32.4 \%$ & 39 & $38.2 \%$ & \\
\hline Comorbidity & & & & & & & .2 \\
\hline Yes & 35 & $34.3 \%$ & 16 & $15.7 \%$ & 19 & $18.6 \%$ & \\
\hline No & 67 & $65.7 \%$ & 23 & $22.5 \%$ & 44 & $43.2 \%$ & \\
\hline Recurrence & & & & & & & .0004 \\
\hline Yes & 46 & $45 \%$ & 9 & $8.8 \%$ & 37 & $36.3 \%$ & \\
\hline No & 56 & $55 \%$ & 30 & $29.4 \%$ & 26 & $25.5 \%$ & \\
\hline Histologic type & & & & & & & .04 \\
\hline Squamous & 58 & $56.9 \%$ & 27 & $26.4 \%$ & 31 & $30.4 \%$ & \\
\hline Nonsquamous & 44 & $43.1 \%$ & 12 & $11.8 \%$ & 32 & $31.4 \%$ & \\
\hline Nodal status & & & & & & & .02 \\
\hline N0-N1 & 73 & $71.5 \%$ & 33 & $32.3 \%$ & 40 & $39.2 \%$ & \\
\hline $\mathrm{N} 2$ & 29 & $28.5 \%$ & 6 & $5.9 \%$ & 23 & $22.6 \%$ & \\
\hline
\end{tabular}

$P A$, Pulmonary artery reconstruction. ${ }^{*}$ Calculated on 99 patients; those undergoing pneumonectomy $(\mathrm{n}=3)$ and tube reconstruction $(\mathrm{n}=3)$ were not included.

respect to the first two variables (feasibility and safety), intraoperative and postoperative management and the prevention and treatment of early complications have been exhaustively addressed in the last decade (Table 3).,18-24 Systemic heparinization has been reduced (1500 unites of heparin intravenously in the last part of our series) to prevent oozing from the sites of mediastinal lymphadenectomy. ${ }^{22}$ Heparin is not reversed with protamine at the end of the operation, and subcutaneous low-molecular weight heparin is administered according to the standard protocols for prevention of pulmonary embolism. Long-term drug administration to prevent clotting is not required if no other disorders coexist. It has also been demonstrated that functional parameters after PA reconstruction do not differ from those currently reported for standard lobectomy. ${ }^{11}$ Echocardiography has shown morphologic and dynamic patterns within the 
TABLE 2. Multivariate analysis performed by Cox regression

\begin{tabular}{lcccc}
\hline \multicolumn{1}{c}{ Variable } & $\$$ & \multicolumn{1}{c}{ SE } & $\boldsymbol{P}$ value & Odds ratio \\
\hline Stage (I-II/III) & 0.2340 & 0.4231 & .580 & \\
Induction (yes/no) & -0.6876 & 0.3427 & .04 & 0.623 \\
Procedure (PA alone/PA with & -0.9798 & 0.2877 & .0006 & 0.382 \\
$\quad$ bronchial sleeve resection) & & & & \\
Complications (yes/no) & 0.3237 & 0.2838 & .254 & \\
Recurrence (yes/no) & 0.6495 & 0.3275 & .04 & 1.524 \\
$\begin{array}{l}\text { Histologic type } \\
\quad \text { nonsquamous/squamous) }\end{array}$ & -0.6616 & 0.2679 & .01 & 0.644 \\
$\quad$ Nodal status (N2/NO-N1) & -0.8948 & 0.3846 & .02 & 0.448 \\
\hline$P A, P U$
\end{tabular}

$P A$, Pulmonary artery reconstruction.

reference range, with normal estimates of PA parameters in $96.2 \%$ of patients. ${ }^{11}$ Thrombosis is an exceptionally rare event when true PA reconstruction is performed $(0.95 \%$ in our series), and an inappropriate tangential resection is avoided when excessive reduction of the lumen is feared.

The oncologic reliability of PA reconstruction remains an important issue to support the value of this procedure. It may be more difficult to demonstrate definitively, however, because randomized trials comparing PA reconstruction with pneumonectomy will probably never be possible. Longterm survival analysis in a large, homogeneous series of patients could certainly help to support this technique. Only a few studies to date have addressed this point, and these have often included heterogeneous populations, including tangential resection and true PA reconstruction (patch, sleeve, conduit). In addition, survival has often been reported in conjunction with bronchial sleeve resection, which does not allow one to draw reliable conclusions.

Our series does not include tangential resection. We consider vascular reconstruction to include only anatomic situations with gaps in the PA surface that must be filled with a patch to avoid stenosis, circumferential resection with end-to-end anastomosis, or conduit interposition. Simple tangential resection has been advocated by other authors when intraoperatively less than a quarter of the anterior wall has to be resected. ${ }^{22,24}$ In such circumstances, we make the same consideration, which also speeds dissection; however, this procedure should not be considered PA reconstruction. Inclusion of tangential resections would have greatly increased our numbers, but outcome analyses would certainly have been less reliable.

Our long-term survival is in line with what can be obtained for patients with NSCLC at similar stages who do not require vascular reconstructions (stage $\mathrm{IB}, 76 \%$ and $44 \%$ at 5 and 10 years; stage II, $52 \%$ and $21 \%$; stage III, $25 \%$ and $12 \%$ ). The overall and disease-free survivals are encouraging, and the local recurrence rate is low; however, the N status, and in particular N2 disease, has a significant effect on outcome, notwithstanding the administration of induction and adjuvant therapy. This is still the most detrimental prognostic factor: $58.6 \%$ of patients with $\mathrm{N} 2$ disease had distant metastases, and only $3 \%$ are alive at 10 years. This aspect was already evident in our previous report ${ }^{11}$; for this reason, the number of patients with $\mathrm{N} 2$ disease has significantly decreased in our practice during the years $(38 \%$ until 1996 and $17 \%$ in the second part of our experience, last 53 cases). Local recurrence was low at only 5.7\% (all patients with stage III NSCLC), confirming, along with the high rate of complete resection, the oncologic value of the procedure. In fact, this local recurrence rate is lower than that seen in previous reports on PA reconstruction ${ }^{24}$ and also lower than the overall rate among patients undergoing standard resection. ${ }^{25} \mathrm{PA}$ reconstruction can be performed safely after induction chemotherapy, in line previously reports for bronchial sleeve resection. In fact, the lower survival observed in this group of patients reflects the stage of the tumor rather than the incidence of postoperative complications.

One apparently new datum that surfaces from this study and is somehow different from our previous report $^{11}$ is that combined bronchovascular reconstructions may offer, from a purely statistical point of view, better survival. This finding might elicit the conclusion that combined reconstructions allow more radical resection. If this were the case, however, there would have been a larger number of local recurrences in the group of patients undergoing isolated PA reconstruction. On the

TABLE 3. Historical results of reconstruction of the pulmonary artery, alone or associated with a bronchial sleeve resection

\begin{tabular}{|c|c|c|c|c|c|}
\hline Reference & Patients & Complications (\%) & Technical complications (\%) & Mortality (\%) & Survival (\% at 5 y) \\
\hline Rendina et al, ${ }^{11} 1999$ & 52 & $13.4 \%$ & $1.9 \%$ & $0 \%$ & $38.3 \%$ \\
\hline Icard et al, ${ }^{23} 1999$ & 16 & NR & NR & NR & $39 \% *$ \\
\hline Okada et $\mathrm{al}^{7}, 2000$ & 21 & NR & $0 \%$ & $0 \%$ & $48 \% *$ \\
\hline Shrager et al, ${ }^{24} 2000 \dagger$ & 33 & $45 \%$ & $0 \%$ & $0 \%$ & $46.7 \% \ddagger$ \\
\hline Fadel et al, ${ }^{18} 2002$ & 11 & $12.4 \%$ & $1.4 \%$ & $0.7 \%$ & $52 \% *$ \\
\hline Chunwei et al, ${ }^{19} 2003$ & 21 & NR & $0 \%$ & NR & $33.3 \% *$ \\
\hline Lausberg et al, ${ }^{20} 2005$ & 67 & NR & $0 \%$ & $1.5 \%$ & $42.9 \% *$ \\
\hline Nagayasu et al, ${ }^{21} 2006$ & 29 & $27.6 \%$ & NR & $17.2 \%$ & $24.2 \% *$ \\
\hline Cerfolio et al, ${ }^{22} 2007$ & 42 & $26 \%$ & $0 \%$ & $2.3 \%$ & $60 \%$ \\
\hline
\end{tabular}

Figures calculated from data reported in articles. $N R$, Not reported. *Overall survival for pulmonary artery and bronchial reconstruction. †Only tangential resections included in this study. †़ Survival at 4 years. 
contrary, the distributions of local recurrence were similar between the groups, with only a slight difference in terms of distant recurrence. The disease-free survival did not show a significant difference between the groups, and a more detailed analysis showed that overall survival of patients undergoing isolated PA reconstruction was mainly affected by nonneoplastic events. This finding was confirmed by univariate and multivariate analyses of disease-free survival.

Overall, PA reconstruction in patients with NSCLC not only is feasible and safe but yields excellent long-term survival. Our results support this technique as a viable and oncologically effective option for patients with NSCLC.

\section{References}

1. Klemper J, Ginsberg RJ. Morbidity and mortality after pneumonectomy. Chest Surg Clin North Am. 1999;9:515-26.

2. Venuta F, Anile M, Diso D, Ibrahim M, De Giacomo T, Rolla M, et al. Operative complications and early mortality after induction chemotherapy for lung cancer. Eur J Cardiothorac Surg. 2007;31:714-7.

3. Venuta F, Sciomer S, Andreetti C, Anile M, De Giacomo T, Rolla M, et al. Longterm Doppler echocardiographic evaluation of the right heart after major lung resections. Eur J Cardiothorac Surg. 2007;32:787-90.

4. Ilonen IK, Räsänen JV, Sihvo EI, Knuuttila A, Sovijärvi AR, Sintonen H, et al. Pneumonectomy: post-operative quality of life and lung function. Lung Cancer. 2007;58:397-402.

5. Alexiu C, Beggs D, Onyeaka P, Kotidis K, Ghosh S, Beggs L, et al. Pneumonectomy for stage I (T1N0 and T2N0) nonsmall cell lung cancer has potent, adverse impact on survival. Ann Thorac Surg. 2003;76:1023-8.

6. Mizushima Y, Noto H, Sugiyama S, Kusajima Y, Yamashita R, Kashii T, et al. Survival and prognosis after pneumonectomy for lung cancer in the elderly. Ann Thorac Surg. 1997;64:193-8.

7. Okada M, Yamagashi H, Stak S, Matsuoka H, Miyamoto Y, Yoshimura M, et al. Survival related to lymph node involvement in lung cancer after sleeve lobectomy compared with pneumonectomy. J Thorac Cardiovasc Surg. 2000;1 19:814-9.

8. Ma Z, Dong J, Fan J, Cheng H. Does sleeve lobectomy concomitant with or without pulmonary artery reconstruction (double sleeve) have favorable results for non-small cell lung cancer compared with pneumonectomy? A meta-analysis. Eur J Cardiothorac Surg. 2007;32:20-8.

9. Ludwig C, Stoelben E, Olschewski M, Hasse J. Comparison of morbidity, 30-day mortality, and long-term survival after pneumonectomy and sleeve lobectomy for non-small cell lung carcinoma. Ann Thorac Surg. 2005;79:968-73.

10. Deslauriers J, Grégoire J, Jacques LF, Piraux M, Guojin L, Lacasse Y. Sleeve lobectomy versus pneumonectomy for lung cancer: a comparative analysis of survival and sites of recurrence. Ann Thorac Surg. 2004;77:1152-6.

11. Rendina EA, Venuta F, De Giacomo T, Ciccone AM, Moretti M, Ruvolo G, et al. Sleeve resection and prosthetic reconstruction of the pulmonary artery for lung cancer. Ann Thorac Surg. 1999;68:995-1002.

12. Rendina EA, Venuta F, De Giacomo T, Vizza DC, Ricci C. Reconstruction of the pulmonary artery by a conduit of autologous pericardium. J Thorac Cardiovasc Surg. 1995;110:867-8.

13. Suen HC, Meyers BF, Guthrie T, Pohl MS, Sundaresan S, Roper CL, et al. Favorable results after sleeve lobectomy or bronchoplasty for bronchial malignancies. Ann Thorac Surg. 1999;67:1557-62.

14. Kawahara K, Akamine S, Takahashi T, Nakamura A, Muraoka M, Tsuji H, et al. Management of anastomotic complications after sleeve lobectomy for lung cancer. Ann Thorac Surg. 1994;57:1529-33.

15. Vogt-Moykopf I, Toomes H, Heinrich S. Sleeve resection of the bronchus and pulmonary artery for pulmonary lesions. Thorac Cardiovasc Surg. 1983;313: 193-8.

16. Read RC, Ziomek S, Ranval TJ, Eidt JF, Gocio JC, Schaefer RF. Pulmonary artery sleeve resection for abutting left upper lobe lesions. Ann Thorac Surg. 1993;55: 850-4.

17. Wada H, Okubo K, Hirata T, Hitomi S. Evaluation of cases with combined bronchoplasty and pulmonary arterioplasty for the treatment of lung cancer. Lung Cancer. 1995;13:113-20.
18. Fadel E, Yildizeli B, Chapelier AR, Dicenta I, Mussot S, Dartevelle PG. Sleeve lobectomy for bronchogenic cancers: factors affecting survival. Ann Thorac Surg. 2002;74:851-9.

19. Chunwei F, Weiji W, Xinguan Z, Qingzen N, Xiangmin J, Qingzhen Z. Evaluation of bronchoplasty and pulmonary artery reconstruction for bronchogenic carcinoma. Eur J Cardiothorac Surg. 2003;23:209-13.

20. Lausberg HF, Graeter TP, Tscholl D, Wendler O, Schäfers HJ. Bronchovascular versus bronchial sleeve resection for central lung tumors. Ann Thorac Surg. 2005; 79:1147-52.

21. Nagayasu T, Matsumoto K, Tagawa T, Nakamura A, Yamasaki N, Nanashima A. Factors affecting survival after bronchoplasty and broncho-angioplasty for lung cancer: single institutional review of 147 patients. Eur J Cardiothorac Surg. 2006;29:585-90.

22. Cerfolio RJ, Bryant AS. Surgical techniques and results for partial or circumferential resection of the pulmonary artery for patients with non-small cell lung cancer. Ann Thorac Surg. 2007;83:1971-7.

23. Icard P, Regnard JF, Guibert L, Magdeleinat P, Jauffret B, Levasseur P. Survival and prognostic factors in patients undergoing parenchymal saving bronchoplastic operation for primary lung cancer: a series of 110 consecutive cases. Eur J Cardiothorac Surg. 1999;15:426-32.

24. Shrager JB, Lambright ES, Mc Grath CM, Wahl PM, Deeb ME, Friedberg JS, et al. Lobectomy with tangential pulmonary artery resection without regard to pulmonary function. Ann Thorac Surg. 2000;70:234-9.

25. Varlotto JM, Recht A, Flickinger JC, Medford-Davis LN, Dyer AM, De Camp MM. Factors associated with local and distant recurrence and survival in patients with resected nonsmall cell lung cancer. Cancer. 2009;115:1059-69.

\section{Discussion}

Dr Shaf Keshavjee (Toronto, Ontario, Canada). Federico, I congratulate you and your team on an excellent report of more than 100 sleeve resections of the PA with an operative mortality as good as or better than most report with simple lobectomy.

First, would you discuss a little further why you think the patients with isolated PA resections did worse than those with combined bronchovascular resections? The 5-year survival was actually half.

Dr Venuta. Thank you for your comments. When this point emerged from the statistical analysis, it really surprised us. We were more than just surprised, we were disappointed. In fact, we feared that this assumption would again open the door to the supporters of pneumonectomy, who believe that the closer we go to the hilum, the better we clean the lymphatic drainage and the higher will be long-term survival. We have also, however, done a diseasefree survival analysis. That disease-free survival is NSCLC-specific survival and shows no significant differences between the groups. These groups show exactly the same incidences of local and distant recurrence. The differences in overall survival between the groups were probably related to the numbers of nonneoplastic events, which were higher in the isolated PA reconstruction population.

Dr Keshavjee. My second question is with respect to oncologic considerations. If you see tumor transgressing the PA wall, that is intraluminally, are you more likely to recommend adjuvant chemotherapy for distant disease concerns?

Dr Venuta. Full-thickness invasion of the vascular wall with tumor inside the artery was extremely rare in our experience. I can clearly remember only 1 patient with a tumor inside the lumen. That patient also had N1 disease and received adjuvant chemotherapy. e believe that this is a rare event that should be addressed on a case-by-case basis with the medical and radiation oncologists.

Dr Keshavjee. Finally, in terms of your decision making, for the patients who might get induction chemotherapy, do you operate to resect the PA on the basis of the original CT findings, or do you 
make your decision to resect the PA intraoperatively on the basis of current findings?

Dr Venuta. The original CT findings certainly give good information about the previous extent of the disease. What we observe on the operating table is much more important, however, and the final decision is made on the basis of these findings.

Dr Mark Krasna (Towson, Md). Dr Venuta, excellent presentation, and we owe you and Dr Rendina a debt in terms of using this technique in our practice.

I have two specific questions. First, you talked about induction therapy. Did any of your patients receive chemotherapy with radiation and then PA resection, or were these cases induction with chemotherapy alone?

Dr Venuta. Our standard policy is to administer induction chemotherapy. All patients in this group received induction chemotherapy, with the exception of 3 who received radiotherapy. Those 3 patients received induction radiotherapy because their disease had been judged inoperable at other institutions, and the radiotherapy was actually administered with therapeutic intent. After our evaluation, these cases were judged operable.

Dr Krasna. And there was no difference between those 3 and the others?

Dr Venuta. Those 3 were the 3 who underwent pneumonectomy under $\mathrm{CPB}$ with patch reconstruction of the origin of the left PA.

Dr Krasna. My other question is related to another technique that you and Dr Rendina have helped us with, which is routine use of the intercostal muscle flap. As you know, we do that after all chemoradiation resections. In my personal practice, I always use the flap as a circumferential wrap when I do the bronchial sleeve. I'm curious, if you're doing a bronchial and arterial sleeve, how do you actually place your intercostal flap?

Dr Venuta. The intercostal flap is always used in case of combined bronchovascular reconstruction, and the flap is wrapped around the bronchus with the technique that we have previously described for isolated bronchial sleeve resection. In such cases, we just pay a little more attention when we pass it between the bronchus and the artery.

Dr Robert J. Cerfolio (Birmingham, Ala). I congratulate you and Dr Rendina. You talked to me about some of the finer techniques of PA resection, and I took those back to Birmingham and have been able to do them, though probably not as well as you. I was a little disappointed that you didn't have more of the actual surgical techniques, because that is the key to this.

I have a couple of quick questions. One of the things you've done is, instead of clamping the PA distally, you have moved that clamp to the inferior pulmonary vein if you're doing a left upper lobe vein. Are you still doing that? Your distal control of the artery is really the vein, allowing you to have a lot of room to work in the PA, correct?

Dr Venuta. Recently we moved to the control of the distal PA at the hilum, if there is enough space to place the clamp. Otherwise, we keep on clamping the inferior vein.

Dr Cerfolio. Well, I love the fact that you taught me how to clamp the vein. It allows me to look inside the artery. It allows me to judge whether there's tension on the artery. Instead of working in a tiny little area in between the artery with a proximal clamp and a distal clamp right next to it, I've got the whole PA wide open and more room and better ability to judge whether I needed to patch or do an end-to-end anastomosis. So I think that's a great trick.

Dr Venuta. That is obviously done when it's possible and there is room to place a clamp. If this is not possible, we are forced to go down to the inferior vein.

Dr Cerfolio. Okay. The second thing is heparin. I have moved away now to using almost no intraoperative heparin. I mean that I will give them 500 units of heparin just to treat "the lawyers," in case there's a clot in the PA, but I don't know whether we really need heparin. Are you heparinizing when you clamp your PA?

Dr Venuta. We use much less heparin than we did before. Now we use between 1500 and 2000 units before clamping. We still don't reverse heparin with protamine at the end of the operation, and these patients receive standard antithrombotic prevention with low-molecular weight subcutaneous heparin, as do all the other patients.

Dr Cerfolio. I'm surprised to hear you say that you wrap the bronchus. I never circumferentially wrap the bronchus. I just put it around the top part of the bronchus. So when I do a PA sleeve with a sleeve of the bronchus and a sleeve of the PA, I just put them in between. Are you really circumferentially wrapping the bronchus or the PA, or just interposing them in between?

Dr Venuta. We wrap the bronchus with the intercostal muscle. In our early studies, we demonstrated that intercostal wrapping contributes to protecting the bronchial anastomosis and separating the potential dehiscence from the rest of the pleural cavity, avoiding bronchopleural or bronchoarterial fistulas. It also contributes to revascularization of the bronchus, and this has been demonstrated with arteriography.

Dr Cerfolio. So obviously since I got every question wrong and you are doing it quite differently from me, I need some more help and learning from you and your group. Thank you. 